\title{
Residual Stress Measurement in Micro-region Using Digital Image Correlation Method
}

\author{
Yanjie Li ${ }^{*}$, Guang Han \\ School of Civil Engineering and Architecture, University of Jinan, Jinan, China
}

Email address:

cea_liyj@ujn.edu.cn (Yanjie Li)

To cite this article:

Yanjie Li, Guang Han. Residual Stress Measurement in Micro-region Using Digital Image Correlation Method. Optics. Special Issue: Optical Techniques for Deformation, Structure and Shape Evaluation. Vol. 4, No. 3-1, 2015, pp. 29-32. doi: 10.11648/j.optics.s.2015040301.17

\begin{abstract}
The residual stress of the zirconia film on a stainless steel substrate is measured using the digital image correlation (DIC) method. A lattice structure is milled by focused ion beam (FIB) and used as the deformation carrier. An annular groove is etched by FIB in order to release the residual stress. The DIC method is used to calculate the deformation caused by the release of residual stress and the residual stress is derived by mechanics equations. The results demonstrate that this method can be extended for micro-region residual stress measurement of other thin films on substrates.
\end{abstract}

Keywords: Digital Image Correlation (DIC), Focused Ion Beam (FIB), Residual Stress

\section{Introduction}

Thin films on a substrate are the most common structure of micro-electro-mechanical systems and thermal barrier coating materials. In the manufacturing process of the films, residual stress is always introduced inevitably. Excessive residual stress can seriously affect the performances of the devices or materials and their service lives. To better understand the generation of residual stress so as to effectively control the magnitude of residual stress, the techniques to measure residual stress need to be developed for film-matrix structures. At present, the existing measurement techniques mainly include X-ray diffraction [1,2], Raman spectroscopy $[3,4]$ and measuring by the release of residual stress[5-12]. For the former two methods, only the average residual stress within the measured area can be obtained. The measurement area is large, generally on the order of millimeters. Furthermore, both methods have some limitations. The X-ray diffraction method is only applicable to crystalline materials, not to amorphous materials. The Raman spectroscopy method is only applicable to materials with the Raman Effect. The methods of releasing residual stress include the drilling method (machining a hole), the grooving method (machining a deep groove) and the ring core method (processing an annular groove). The ring core method not only applies to macroscopic residual stress measurement, but also to microscopic residual stress measurement, and is applicable to a variety of different materials. Since the destruction of films is often due to local damage caused by the residual stress, it is of important significance to measure residual stress at a micro-region.

The focused ion beam (FIB) system with micro-nano machining ability combined with optical measurement method offers the potential of the residual stress measurement at the micro area. Sabate et al. [5-7] used FIB to fabricate a hole or a slot at the film surface to release the residual stress and utilized digital image correlation (DIC) to measure residual stress in a film. Massl et al. [8] used FIB to process micro cantilever and measured its deflection by DIC to obtain the residual stress of a film. Korsunsky, Sebastiani and Zhu et al.[9-12] used FIB to process annular grooves and utilized DIC method to measure the residual stress of a film. A hole or a groove will cause strain concentration at the etched area. The micro-cantilever processing method is only applicable to the edge of the specimen, and only applies to a film on a brittle substrate. In comparison, the strain released by the ring core method is relatively uniform. Therefore it is a favorable choice to release residual stress. This paper will use DIC combined with the ring core method by FIB processing to measure micro-scale residual stress of the zirconia film on a stainless steel substrate.

\section{Principle of Digital Image Correlation Method}

A two-dimensional DIC measuring system is shown in Figure 1. The tested plane specimen is placed in the loading 
system so that the optical axis of image acquisition device is vertical to the specimen surface. Real-time speckle patterns on specimen surface are recorded under different magnitudes of the load, which requires the imaging surface of the specimen with random grayscale distribution. Typically the image before loading is denoted as "reference image" and the rest of the individual images are called as "deformed images". The image acquisition device may be a CCD camera, may also be a synchronized high-speed camera, an optical microscope, a scanning electron microscope (SEM), a laser scanning confocal microscope (LSCM), a scanning tunneling microscope (STM), and an atomic force microscope (AFM).

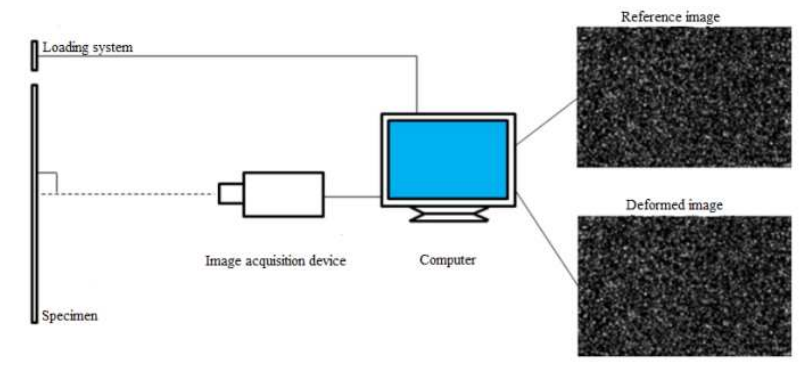

Figure 1. Two-dimensional DIC measurement system.

Correlation calculation between the images acquired before and after deformation is carried out to find out corresponding points before and after deformation so that the deformation of the specimen surface can be derived.

The basic principle of two-dimensional DIC is shown in Figure 2. Take the tested point $\left(x_{0}, y_{0}\right)$ in the reference image $R(x, y)$ as the center and a square with dimensions of $(2 M+1) \times(2 M+1)$ pixels as a reference subset. In the image after deformation $D\left(x^{\prime}, y^{\prime}\right)$, correlation calculation is carried out in accordance with a certain search strategy and the correlation function, in order to find out the most correlated target subset with the reference subset in the reference image. Assuming its center is $\left(x_{0}{ }^{\prime}, y_{0}{ }^{\prime}\right)$, the displacement components in the $x$ and $y$ directions, i.e. $u$ and $v$, are $x_{0}{ }^{\prime}-x_{0}$ and $y_{0}{ }^{\prime}-y_{0}$, respectively.
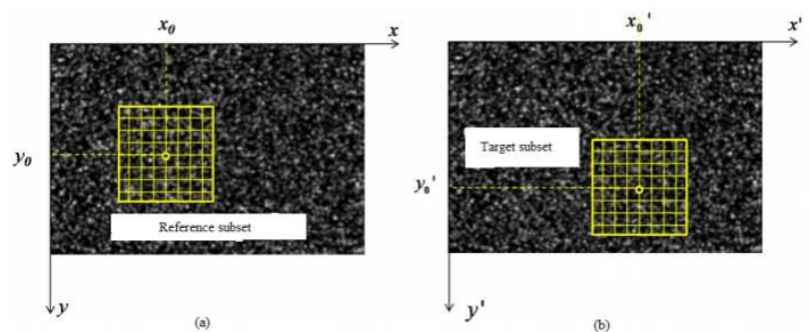

Figure 2. The schematic of subsets before and after deformation.

A correlation function to evaluate the correlation degree between the subsets in the image before and after deformation is defined as:

$$
C_{R, D}(\vec{p})=\operatorname{Corr}\left\{R(x, y), D\left(x^{\prime}, y^{\prime}\right)\right\}
$$

where $R(x, y)$ is the grayscale of point $(x, y)$ in the reference image, $D\left(x^{\prime}, y^{\prime}\right)$ is the grayscale of the target point $\left(x^{\prime}, y^{\prime}\right)$ in the image after deformation, and Corr is the correlation function describing the correlation between $R(x, y)$ and $D(x$ ', $\left.y^{\prime}\right)$. There are various expression forms of the correlation function. Among others, the normalized cross-correlation function, the normalized minimum squared distance, and the minimum squared distance with parameters are most commonly used because they are insensitive to the linear variation of grayscale in the subset. Besides selecting the correlation function, the size of the subset, the calculation step and sub-pixel algorithms should also be determined before the correlation calculation.

\section{The Measuring Principle of Micro-region Residual Stress}

Specimen images are recorded before and after the release of residual stress. DIC is used to calculate the displacement of the specimen due to the release of residual stress. Then the strain $\varepsilon_{x}, \varepsilon_{y}$ and $r_{x y}$ can be obtained, thus the principal strains are as follows

$$
\left\{\begin{array}{l}
\varepsilon_{1}=\frac{1}{2}\left[\left(\varepsilon_{x}+\varepsilon_{y}\right)+\sqrt{\left(\varepsilon_{x}-\varepsilon_{y}\right)^{2}+r_{x y}^{2}}\right] \\
\varepsilon_{2}=\frac{1}{2}\left[\left(\varepsilon_{x}+\varepsilon_{y}\right)-\sqrt{\left(\varepsilon_{x}-\varepsilon_{y}\right)^{2}+r_{x y}}\right]
\end{array}\right.
$$

The two principal residual stresses are

$$
\left\{\begin{array}{l}
\sigma_{1}=-\frac{E}{1-v^{2}}\left(\varepsilon_{1}+v \varepsilon_{2}\right) \\
\sigma_{2}=-\frac{E}{1-v^{2}}\left(\varepsilon_{2}+v \varepsilon_{1}\right)
\end{array}\right.
$$

where $E$ is the elastic modulus, which can be measured by nano-indentation or other techniques, and $v$ is the Poisson's ratio.

\section{Experiments of Residual Stress Measurement of Micro-region}

\subsection{Preparation of Tested Specimen}

The tested object is a zirconia coating film $\left(8 \% \mathrm{wt} \mathrm{Y}_{2} \mathrm{O}_{3}\right)$ on a substrate of stainless steel. The thickness of the film is approximately $500 \mu \mathrm{m}$, as shown in Figure 3. It is made by thermal spraying process as follows:

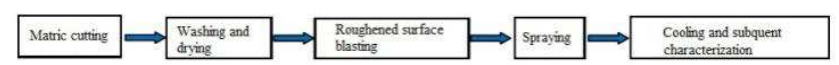

Before spraying on the specimen, surface blasting treatment can improve the surface roughness to enhance the interfacial adhesion. Blasting and spraying parameters are listed in Table 1. The adhesive layer is NiCrCoAlY nickel-based alloy powder.

In order to create a smooth surface so as to facilitate the 
processing of FIB, the polishing treatment is conducted to the specimen surface. Firstly, the specimen is polished with sandpaper, and then polished with polishing paste. Since zirconia is non-conductive, a thin layer of gold film is coated on the specimen surface prior to the experiment.

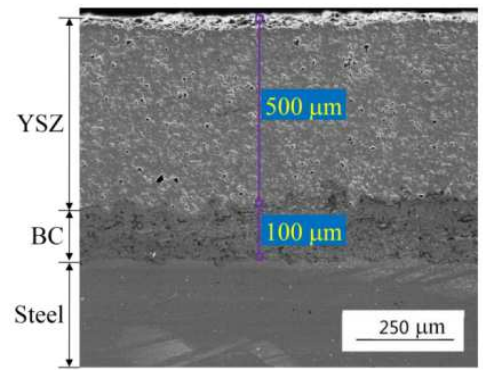

Figure 3. Schematic of the zirconia film on a stainless steel substrate.

Table 1. Blasting and spraying parameters.

\begin{tabular}{lll}
\hline & Voltage & $70 \mathrm{~V}$ \\
Spraying & Electric current & $500 \mathrm{~A}$ \\
& Spraying distance & $125 \mathrm{~mm}$ \\
& Gun Speed & $300 \mathrm{~mm} / \mathrm{s}$ \\
& Powder feed rate & $30 \mathrm{~g} / \mathrm{min}$ \\
Blasting & Material & Corundum \\
& Diameter & $4 \mathrm{~mm}$ \\
& Pressure & $0.7 \mathrm{MPa}$ \\
\hline
\end{tabular}

\subsection{Experiment Process}

This experiment is carried out in the FIB - field emission SEM double beam system (Tescan Lyra3). The specimen is stuck on the sample stage with 502 glue, and a conductive tape is used to connect the sample surface and the sample stage in order to discharge. The specimen and the sample stage are placed in the vacuum chamber, in which case the electron beam is perpendicular to the specimen surface. After the vacuum, the electron beam alignment, anastigmatism and focusing are carried out until the specimen surface can be clearly observed so as to select the measurement zone of residual stress. Because of the $55^{\circ}$ angle between the electron beam and the ion beam, the specimen needs to be tilted $55^{\circ}$ so that the ion beam is perpendicular to the specimen surface. The ion beam alignment, anastigmatism and focusing are then carried out in order to locate the tested area, whose size is 2 $\mu \mathrm{m} \times 2 \mu \mathrm{m}$. In this region speckle lattice is fabricated using the FIB and the lattice period is $130 \mathrm{~nm}$. Then the specimen is inclined $55^{\circ}$ so that it is returned to the position when the electron beam is perpendicular to the specimen surface to record speckle dots, as shown in Figure 4(a). Then the test specimen is inclined $55^{\circ}$ so that the ion beam is perpendicular to the specimen surface to release residual stress by etching an annular groove with FIB. The inner diameter of the annular groove is $2 \mu \mathrm{m}$, the outer diameter is $4 \mu \mathrm{m}$, and the depth is 2 $\mu \mathrm{m}$. Its shape is shown in Figure 5. After completion of the etching of the annular groove, the specimen is inclined $55^{\circ}$ so that it is returned to the position when the electron beam is perpendicular to the specimen surface to record speckle dots, as shown in Figure 4 (b).

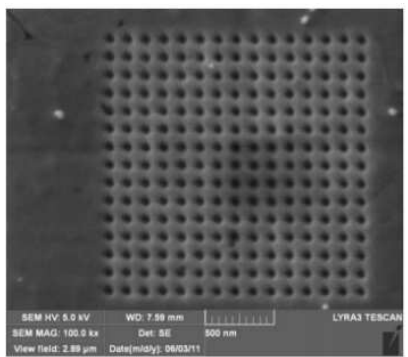

(a) Before etching the annular groove

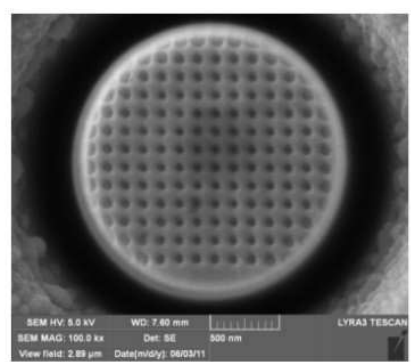

(b) After etching the annular groove
Figure 4. Speckle lattice structure etched around the annular groove.

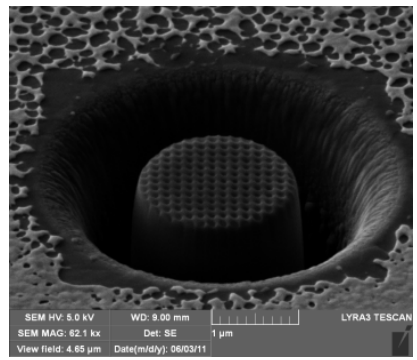

Figure 5. Annular groove morphology.

\section{The Experimental Results}

The speckle image after etching annular groove is used as the reference image, while the speckle image before etching annular groove is used as the image after deformation. This calculation sequence is due to that the speckle region before etching the annular groove is greater than the speckle region after etching the annular groove, which makes the calculation more accurate. The displacement field and then the strain field caused by the residual stress release are calculated using the DIC method, as shown in Figure 6. The average normal strain and the shear strain are as follows:

$$
\varepsilon_{x}=-3471 \mu \varepsilon, \varepsilon_{y}=-7049 \mu \varepsilon, r_{x y}=804 \mu \varepsilon
$$

Therefore the principal strains are as follows:

$$
\begin{aligned}
& \varepsilon_{1}=\frac{1}{2}\left[\left(\varepsilon_{x}+\varepsilon_{y}\right)-\sqrt{\left(\varepsilon_{x}-\varepsilon_{y}\right)^{2}+r_{x y}{ }^{2}}\right]=-7094 \mu \varepsilon \\
& \varepsilon_{2}=\frac{1}{2}\left[\left(\varepsilon_{x}+\varepsilon_{y}\right)+\sqrt{\left(\varepsilon_{x}-\varepsilon_{y}\right)^{2}+r_{y y}{ }^{2}}\right]=-3246 \mu \varepsilon
\end{aligned}
$$

In fact the strain of the specimen after etching the annular groove relative to that before the etching is required. There is a relationship between the actual strain $\mathcal{E}_{a}$ and the measured strain $\mathcal{E}_{m}$ :

$$
\varepsilon_{a}=\frac{1}{1+\varepsilon_{m}}-1
$$

Thus the actual principal strains are

$$
\begin{aligned}
& \varepsilon_{1 a}=7144 \mu \varepsilon \\
& \varepsilon_{2 a}=3438 \mu \varepsilon
\end{aligned}
$$

The elastic modulus of the zirconia coating film $E$ is 137 
GPa, as measured by the nano-indentation technique, and the Poisson's ratio $v$ is 0.3 . Thus both principal residual stresses are as follows:

$$
\begin{aligned}
& \sigma_{1}=-\frac{E}{1-v^{2}}\left(\varepsilon_{1}+v \varepsilon_{2}\right)=-1234 \mathrm{MP} a \\
& \sigma_{2}=-\frac{E}{1-v^{2}}\left(\varepsilon_{2}+v \varepsilon_{1}\right)=-843 \mathrm{MP} a
\end{aligned}
$$
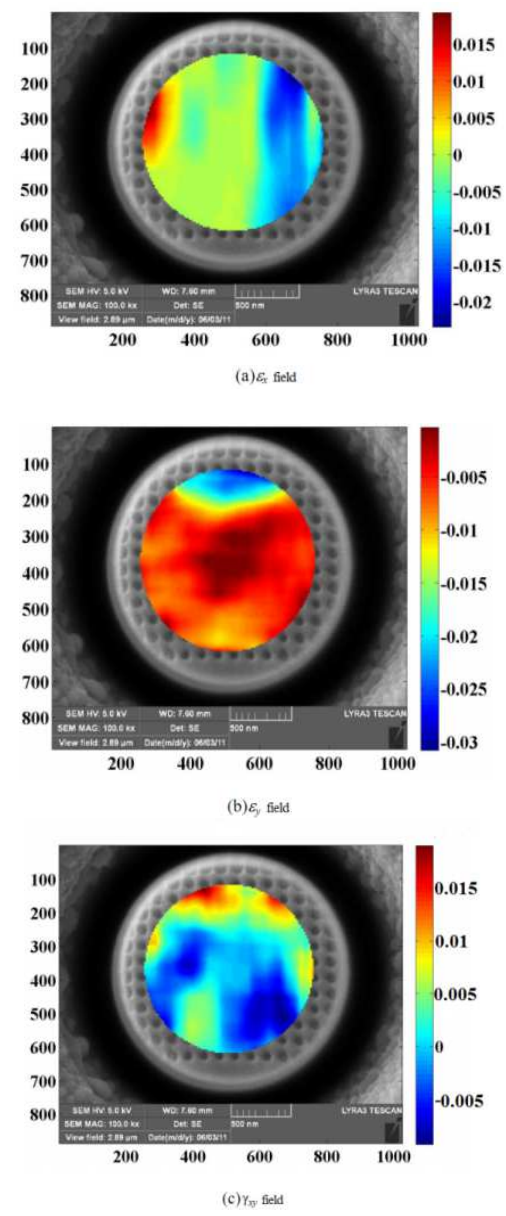

Figure 6. The strain field caused by the release of residual stress.

\section{Summary}

By combining DIC method and ring core method, we have measured the residual stress of the zirconia film on the stainless steel substrate. A periodical lattice structure is used as the deformation carrier. Since the lattice on the region nearby the groove will be damaged during the FIB milling process, the measurement accuracy in this region will be influenced, therefore protection practice will be conducted in the next step. Furthermore, in order to enhance the measurement precision, random speckle patterns will be fabricated and used as the deformation carrier.

\section{Acknowledgements}

The authors are grateful to the financial support from the National Natural Science Foundation of China (Grant No.11302082), and the Doctoral Program of University of Jinan (XBS1307).

\section{References}

[1] Tang W, Deng L J, Xu K W, et al. X-ray diffraction measurement of residual stress and crystal orientationin $\mathrm{Au} / \mathrm{NiCr} / \mathrm{Ta}$ films prepared by plating. Surface \& Coatings Technology, 2007, 201:5944-5947.

[2] Yang F, Fei W D, Gao Z M, et al. An alternative micro-area $X$-ray diffraction method for residual stress measurement of $\mathrm{Pb}(\mathrm{Zr}, \mathrm{Ti}) \mathrm{O} 3$ film. Surface \& Coatings Technology, 2007, 202: 121-125.

[3] Kang Y L, Qiu Y, Lei Z K, et al. An application of Raman spectroscopy on the measurement of residual stress in porous silicon.Optics and Lasers in Engineering, 2005, 43: 847-855.

[4] Zhu W L, Zhu J L, Nishino S, et al. Spatially resolved Raman spectroscopy evaluation of residual stresses in $3 \mathrm{C}$-SiC layer deposited on $\mathrm{Si}$ substrates with different crystallographic orientation. Applied Surface Science, 2006, 252: 2346-2354.

[5] Sabate N, Vogel D, Gollhardt A, et al. Residual stress measurement on a MEMS structure with high-spatial resolution. Journal of Microelectromechanical Systems, 2007, $16(2): 365-372$.

[6] Sabate N, Vogel D, Gollhardt A, et al. Digital image correlation of nanoscale deformation fields for local stress measurement in thin films. Nanotechnology, 2006, 17(20):5264-5270.

[7] Sabate N, Vogel D, Gollhardt A, et al. Measurement of residual stresses in micromachined structures in a microregion. Applied physics letters, 2006, 88:071910.

[8] Massl S, Keckes J, Pippan R. A new cantilever technique reveals spatial distributions of residual stresses in near-surface structures. Scripta materialia, 2008, 59(5):503-506.

[9] Korsunsky A, Sebastiani M,Bemporad E. Residual Stress Evaluation at the Micrometer Scale: Analysis of Thin Coatings by FIB milling and Digital Image Correlation. Surface and Coatings Technology, 2010, 205(7):2393-2403.

[10] Korsunsky A, Sebastiani M, Bemporad E. Focused ion beam ring drilling for residual stress evaluation. Materials Letters, 2009, 63(22):1961-1963.

[11] Sebastiani M, Eberl C, Bemporad E, et al. Depth-resolved residual stress analysis of thin coatings by a new FIB-DIC method. Materials Science \& Engineering A, 2011, 528(27):7901-7908.

[12] Zhu J G, Xie H M, Li Y J, et al. Interfacial Residual Stress Analysis of Thermal Spray Coatings by Miniature Ring-Core Cutting Combined with DIC Method. Experimental Mechanics, 2014, 54(2): 127-136. 